\title{
A Biomolecular Sensing Platform Using RF Active System
}

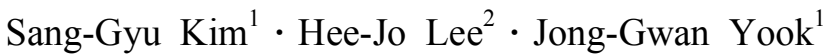

\begin{abstract}
This paper describes a novel and compact biosensing platform using an RF active system. The proposed sensing system is based on the oscillation frequency deviation due to the biomolecular binding mechanism on a resonator. The impedance variation of the resonator, which is caused by a specific biomolecular interaction results in a corresponding change in the oscillation frequency of the oscillator so that this change is used for the discrimination of the biomolecular binding, along with concentration variation. Also, a Surface Acoustic Wave (SAW) filter is utilized in order to enhance the biosensing performance of our system. Because the oscillator operates at the skirt frequency range of the SAW filter, a small amount of oscillation frequency deviation is transformed into a large variation in the output amplitude. Next, a power detector is used to detect the amplitude variation and convert it to DC voltage. It was also found that the frequency response of the biosensing system changes linearly with three streptavidin concentrations. Therefore, we expect that the proposed RF biosensing system can be applied to bio/medical applications capable of detecting a nano-sized biomolecular interaction.
\end{abstract}

Key words: Biomolecule, Biosensor, Oscillator, SAW filter.

\section{I . Introduction}

In recent years, RF biosensing techniques have been received much attention due to the fact that they provide label-free, non-destructive, and non-invasive detection [1], [2]. From these features, various RF biosensing schemes, such as single-walled carbon nanotubes (SWNTs), meta-materials (MTMs), and so on, were introduced in order to detect the specific biomolecules and cells [3] [6]. For example, RF resonator-based biosensors were studied for human cell detection via a micro-fabrication method [7] [9]. Although these methods explored in these research projects were simple and high sensitive for biomolecular sensing, they still have disadvantages in that they require very expensive and complex equipment, including an RF probe station associated with a Vector Network Analyzer (VNA), as well as the micro-fabrication process. Moreover, all of these resonance-based methods require several resonator arrays, along with a high-Q resonator in order to enhance the sensitivity. However, these biosensing systems not only occupy a large area but also consume a large sample. Moreover, it has been revealed that the frequency change for small biomolecular quantities is quite small. As a result, the sensitivity of a conventional RF biosensing scheme is limited to the detection of very low biomolecular concentrations.

In this work, an RF active system based on oscillation frequency deviation for small biomolecule detection is proposed at the $2.4 \mathrm{GHz}$ Industrial, Science, and Medical (ISM) band. The basic concept of the proposed system is that when specific biomolecules are bound to the gold surface on a resonator, the resonant frequency of the oscillator changes due to the impedance variation of the resonator. Based on the concept of the RF biosensing system, the feasibility as a biosensing system for rapid and label-free biomolecular detection is experimentally demonstrated.

\section{Operating Principle and System Configuration}

\section{2-1 Operating Principle}

Fig. 1 shows the architecture of the proposed RF biosensing system. The system consists of an oscillator with a gold-coated resonator, a SAW filter, and a power

\footnotetext{
Manuscript received October 22, 2012 ; Revised November 15, 2012 ; Accepted November 28, 2012. (ID No. 20121022-01J)

${ }^{1}$ School of Electrical and Electronic Engineering, Yonsei University, Seoul, Republic of Korea.

${ }^{2}$ School of Mechanical Engineering, Yonsei University, Seoul, Republic of Korea.

Corresponding Author : Jong-GwanYook (e-mail : jgyook@yonsei.ac.kr)
}

This is an Open-Access article distributed under the terms of the Creative Commons Attribution Non-Commercial License (http://creativecommons.org/licenses/ by-nc/3.0) which permits unrestricted non-commercial use, distribution, and reproduction in any medium, provided the original work is properly cited. 


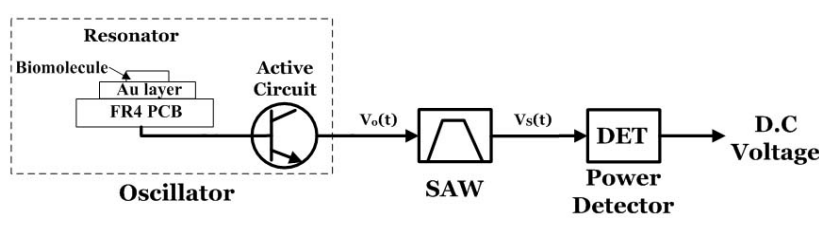

Fig. 1. Proposed biosensing system architecture.
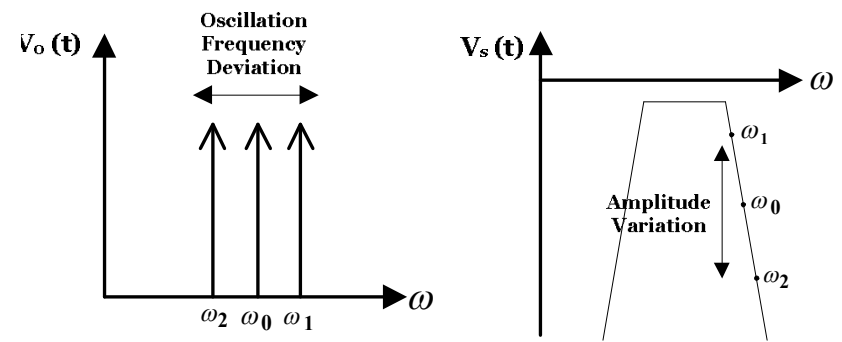

(a) Oscillator output spectrum

(b) SAW filter output spectrum

Fig. 2. Output spectrum.

detector. The detection principle is based on the oscillation frequency deviation, along with the impedance variation, which is caused by biomolecular binding on the gold surface. In particular, the planar resonator has been used as a sensor, as well as a series feedback component, in this system. When biomolecules are bound to the gold surface, the input impedance may change. This is due to variations in the capacitive and inductive components of the resonator. It is assumed that the impedance variation and resulting frequency shifts are in the locking range of the oscillator and this variation is eventually utilized for biosensing, as illustrated in Fig. 2 (a). Since the biomolecular size is just a few nano-orders, the frequency deviation due to the biomolecular binding effect may be too small for detection, so an RF SAW filter is utilized in order to enhance the sensitivity of the system. This is based on fact that the amplitude of the SAW filter output considerably changes in the skirt frequency range. As a result, a small frequency deviation in the oscillation frequency can be transformed into a large output amplitude variation by the SAW filter, as illustrated in Fig. 2 (b). In the last stage, an RF power detector is adapted in the output of the SAW filter in order to transform the frequency variation of the oscillator into the variation of DC voltage, which can be easily measured with convenient equipment, such as a digital multi-meter.

\section{2-2 Design of Sensing Parts}

Fig. 3(a) shows the design and dimension of a resonator. The dielectric constant and height of the substrate used are 4.4 and $1 \mathrm{~mm}$, respectively. The conductors of

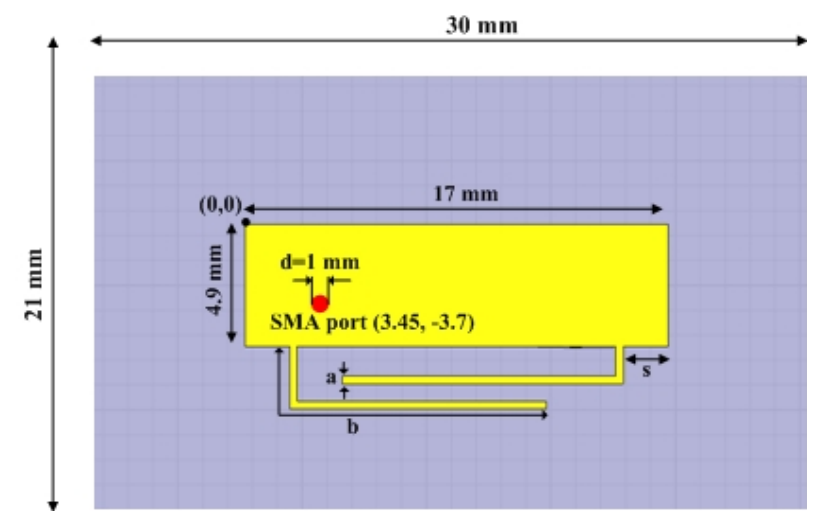

(a) Shape and dimensions of the resonator

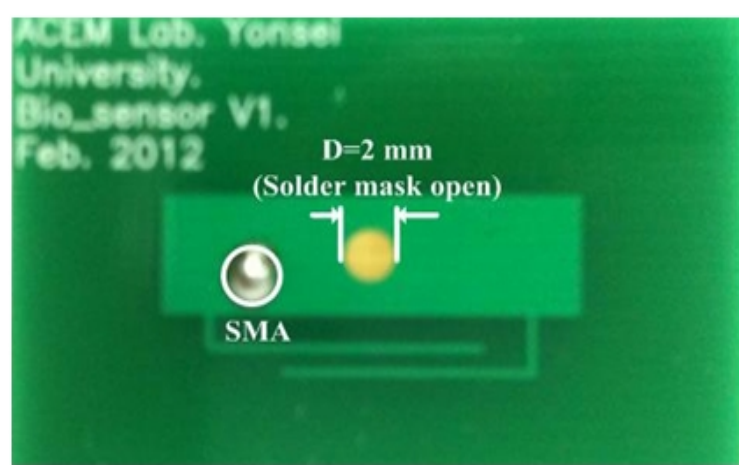

(b) Fabricated sample

Fig. 3. Design and fabrication of the proposed resonator.

the top (resonator) and bottom (ground plane) sides are coated with gold. The resonator has a small rectangle $17 \mathrm{~mm}$ I length and $4.9 \mathrm{~mm}$ in width. Two similar arms of dimensions $a=0.3 \mathrm{~mm}$ and $b=12.2 \mathrm{~mm}$ are placed symmetrically on the corners of the rectangle at an offset of $s=0.3 \mathrm{~mm}$. The overall size of the resonator, including the ground plane, is $30 \mathrm{~mm}$ by $21 \mathrm{~mm}$. Fig. 3(b) shows the fabricated sample, where the top side is covered with a solder mask, and a circular area with a

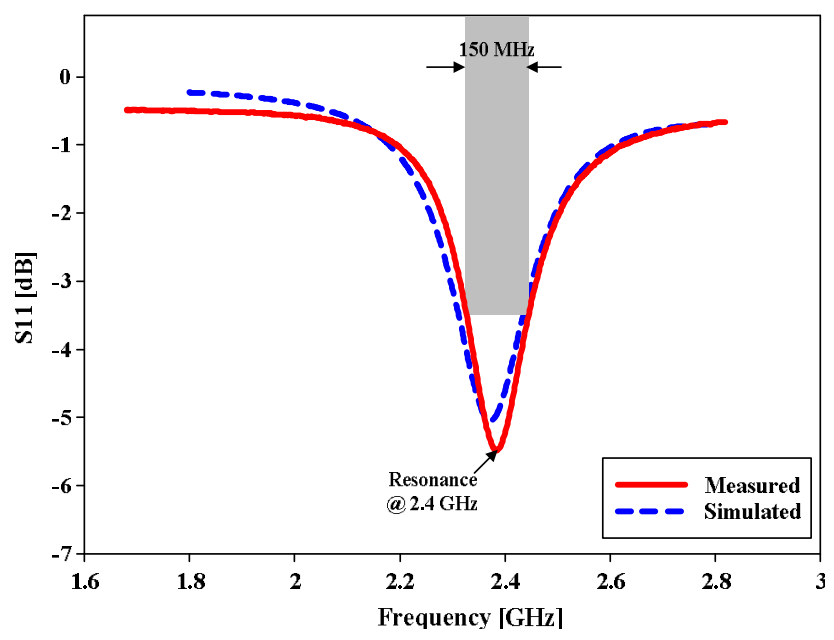

Fig. 4. Measured and simulated results of the resonator. 


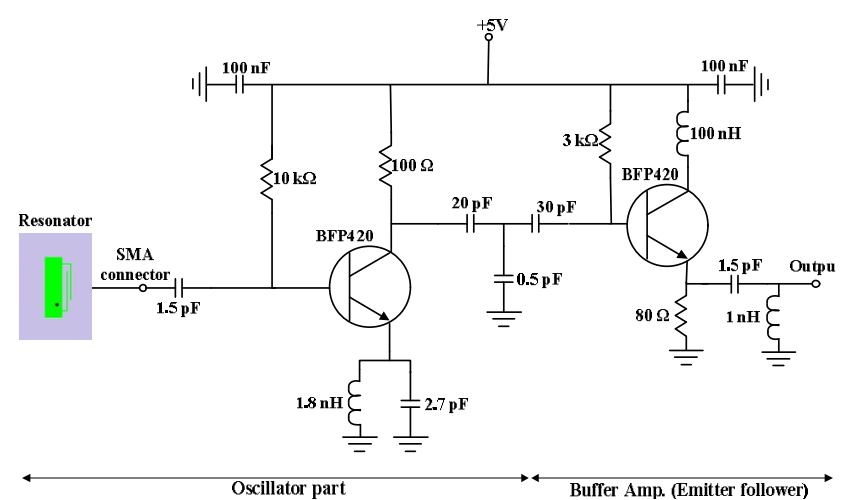

Fig. 5. Schematic of the oscillator with a resonator and buffer amplifier.

diameter of $2 \mathrm{~mm}$ is opened in order to detect a biomolecule.

To predict the resonant characteristics of the resonator, a simulation was performed with a 3D full-wave electromagnetic solver (Ansoft HFSS), and the simulation results were in good agreement with the measured results, as shown in Fig. 4. The resonance occurs at 2.4 $\mathrm{GHz}$ with a 3-dB bandwidth of $150 \mathrm{MHz}$; thus, the loaded $Q$ value is about 15. Conventionally, a high-Q resonator is used in oscillator designs in order to improve the frequency stability and phase noise characteristics [10]. However, a low-Q resonator is preferable to a high-Q resonator in this application due to the reduced frequency stability requirements. Suitable impedance control around a bipolar transistor can be achieved with an oscillation condition at the resonant frequency of the resonator [11]. Fig. 5 shows a schematic diagram of the $2.4 \mathrm{GHz}$ amplifier and the cascade type resonator combined with an emitter follower-type buffer amplifier, in which the resonator simultaneously functions as a sensor and a feedback element. The design theory of this oscillator is already well-developed [12]. Here a BFP 420 npn silicon RF transistor is used as an active device. When the SAW filter is directly connected to the oscillator, close attention must be paid because the oscillation condition can be changed by the rapid impedance variation at the skirt frequency range of the SAW filter. For this reason, the buffer amplifier of the emitter follower-type is used to prevent a strong mutual interaction between the oscillator and the filter. A PCB that was $100 \mathrm{~mm}$ by $100 \mathrm{~mm}$ in size was fabricated, which is a part of the system part, as shown in Fig. 6. Both PCBs, the resonator part, shown in Fig. 3(b), and the system part, are connected through an SMA connector. Consequently, the measured oscillator output power is $2 \mathrm{dBm}$ at $2.517 \mathrm{GHz}$, including the output of the emitter follower buffer amplifier, as shown in Fig. 7.

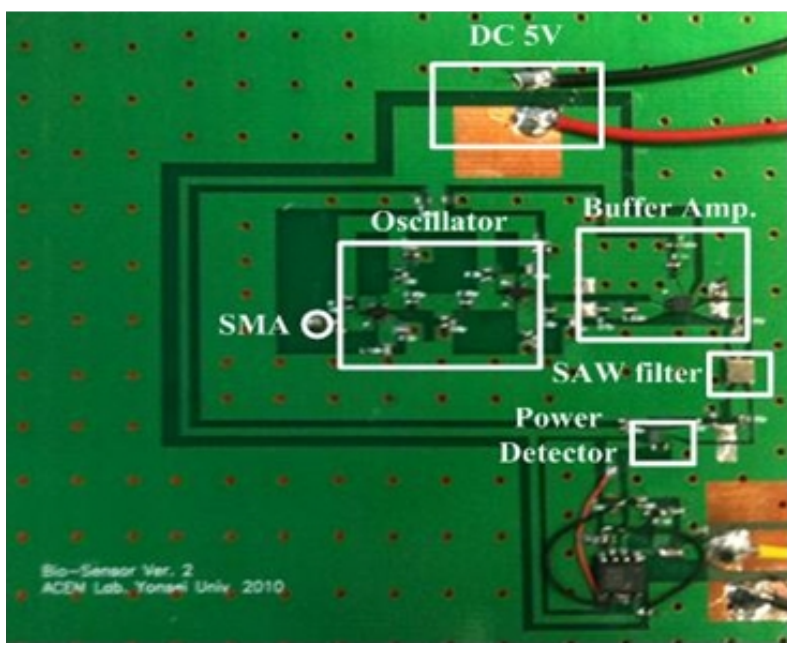

Fig. 6. Fabricated system part PCB.

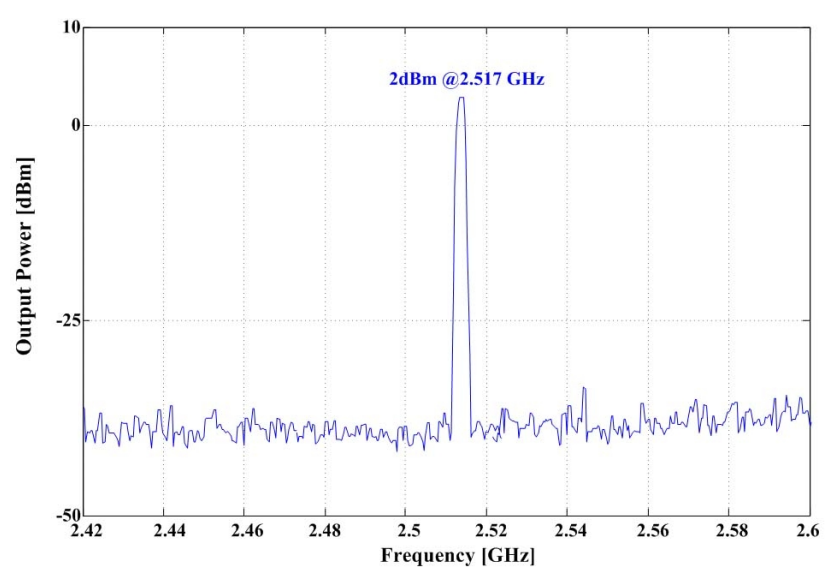

Fig. 7. Measured output power spectrum of the oscillator.

Meanwhile, when the biomolecule is bound to the gold layer of the resonator, the conductivity of the gold surface is changed according to the concentration of the biomolecules. The variation in the conductivity causes a variation in the resonator impedance, and the oscillation frequency is consequently changed. A Surface Acoustic Wave (SAW) filter is used to convert frequency deviation to amplitude variation. When the oscillator operates at the skirt frequency range $(2.49 \sim 2.54 \mathrm{GHz})$ of the SAW filter, a small amount of oscillation frequency deviation can be transformed to a large variation in the amplitude variation.

Fig. 8 shows the measured performance of the SAW filter used, which is an SA2441AM from SAWNICS. The designed oscillator operates at $2.517 \mathrm{GHz}$ in the skirt frequency range of the SAW filter, and the average rate of change for a magnitude variation of $1 \mathrm{~dB} / \mathrm{MHz}$ can be achieved. Assuming the frequency deviation caused by biomolecular binding is about $1 \mathrm{MHz}$, the expected amplitude variation of the SAW filter is $1 \mathrm{~dB}$. 


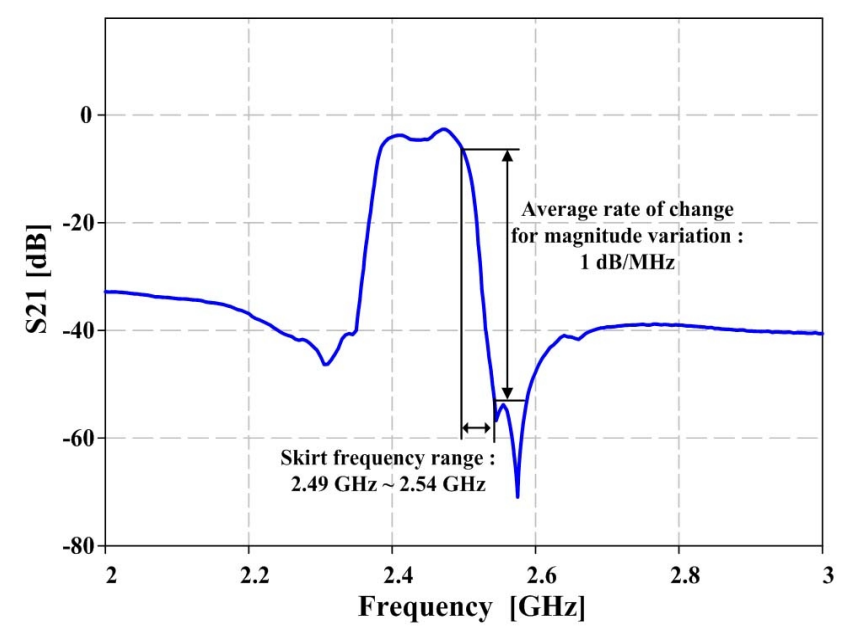

Fig. 8. Measurement results of the SAW filter's performance.

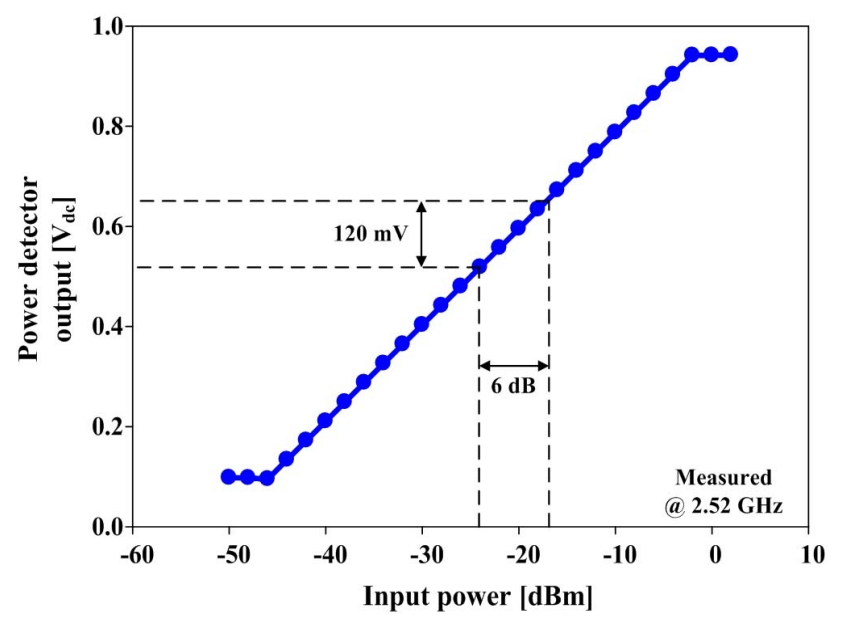

Fig. 9. Measurement results of power detector output at $2.52 \mathrm{GHz}$.

Thus, it enables a small frequency deviation to be transformed into a large amplitude variation of the output voltage. The amplitude variation is then detected by an RF power detector. An AD8312 RF detector from Ana$\log$ Devices Inc. is used. It has a frequency response that ranges from $50 \mathrm{MHz}$ to $3.5 \mathrm{GHz}$ and a detection power level that ranges from -45 to $0 \mathrm{dBm}$, and its measurement results are shown in Fig. 9.

\section{Biomolecular Processing}

Fig. 10 shows a biological process for binding between thiols-linked biotin and streptavidin in order to verify the feasibility of the biomolecular sensing device. First of all, the biotinylated thiols are used as a simple and direct construction for immobilization on the gold surface. Here, a thiols (-SH) substrate can enhance the

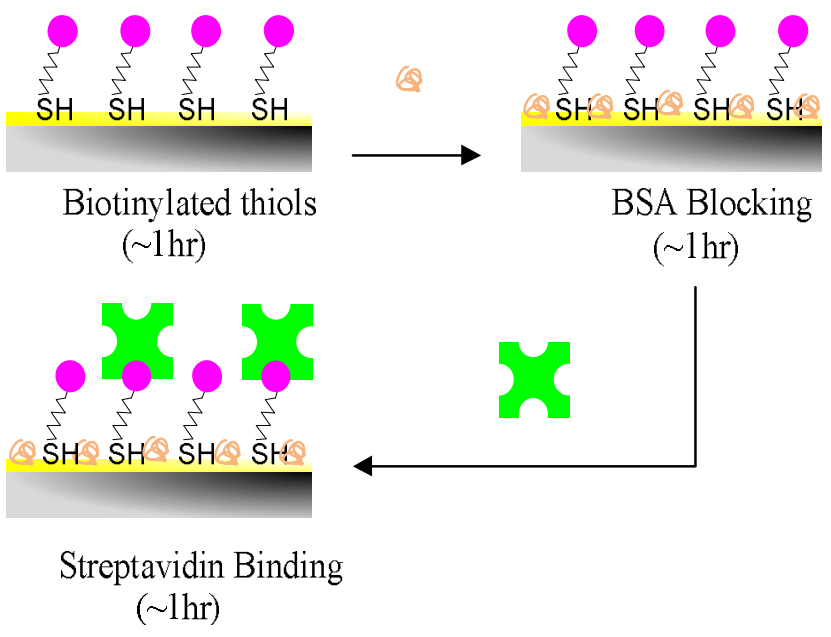

Fig. 10. Biotin-streptavidin binding system.

sensitivity and selectivity, and can specify a chemically modified gold surface; therefore, it has been widely used as a biological receptor of many gold-based biosensors $[13] \sim[15]$.

The biotin (a vitamin series) used has a strong chemical affinity for the biomolecules and streptavidin (a protein series). In this work, the biological process has been carried out as follows. First, all samples are simultaneously immobilized with a $25 \mu \mathrm{g} / \mathrm{ml}$ concentration of biotinylated thiols in deionized (DI) water for about 1 hour. Next, to deactivate and block the excess reactive groups remaining on the surface, the devices are treated with Bovine Serum Albumin (BSA) of at a concentration of $25 \mu \mathrm{g} / \mathrm{ml}$ for about 1 hour. Finally, the biotin was coupled with three streptavidin concentration, i.e. 100,10 , and $1 \mathrm{ng} / \mathrm{ml}$ in DI water for about 1 hour. After the samples are washed with pure DI water, the dried samples are measured with the RF measurement system.

\section{Measurement Results}

The proposed biosensing system was designed and fabricated on the basis of the sensing mechanism, as mentioned in Section II. The measurement setup consists of the proposed sensing system, power supply, spectrum analyzer, and digital multi-meter. The detectable resonator is connected to the proposed sensing supply voltage and the operating currents are $6 \mathrm{~V}$ and $75 \mathrm{~mA}$, respectively. The spectrum analyzer is used to confirm the oscillation frequency deviation, and the final output of the proposed sensing system, which is DC voltage, is measured via a digital multi-meter. Before starting the biological process, the deionized water (DI) solution on the resonator is tested. Three samples are prepared, and 


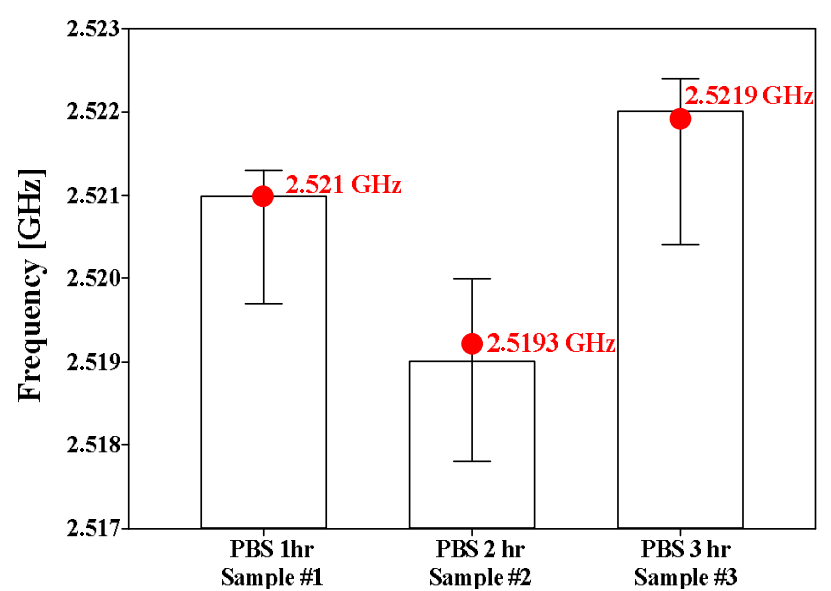

Fig. 11. DI solution test for three bare samples.

each sample is measured five times for oscillation frequency deviation. The range of the measurement result is presented as the maximum, average, and minimum values. After a thorough washing process, samples reveal slight differences from the bare samples for all three configurations, i.e., 1-, 2-, and 3- hour immersion cases, as shown in Fig. 11. However, the discrepancy is not a critical problem because the frequency shift is within the oscillation frequency range of each sample. This means that the DI solution to be used for the biological process would cause only a minimal effect on oscillation frequency deviation. Also, fifteen bare samples are prepared for the biological process, and each sample is measured five times. The measurement items are oscillation frequency deviation, SAW filter output power, and the power detector output voltage for three streptavidin concentrations $(100 \mathrm{ng} / \mathrm{ml}, 10 \mathrm{ng} / \mathrm{ml}$, and 1 $\mathrm{ng} / \mathrm{ml}$ ). Fig. 12(a) shows the deviation of the oscillation frequency for the thiols-linked biotin, as well as for the three different streptavidin concentrations. It is found that the frequency deviation clearly exhibits linear shifts with streptavidin concentrations. This linear variation is observed in the SAW filter output power, as well as the power detector output voltage, as summarized in Figs. 12(b) and 12(c), respectively. The results show that the frequency shift after the immobilization of the biotinylated thiols is smaller than those of the streptavidin binding. This is because the biotin exists in a smaller biomolecular quantity than the streptavidin. Thus, it is clearly demonstrated that the proposed low-Q resonatorbased oscillator and detection scheme can detect the biomolecular binding process at the $1 \mathrm{ng} / \mathrm{ml}$ concentration level.

\section{Conclusion}

An RF sensing system based on oscillation frequency

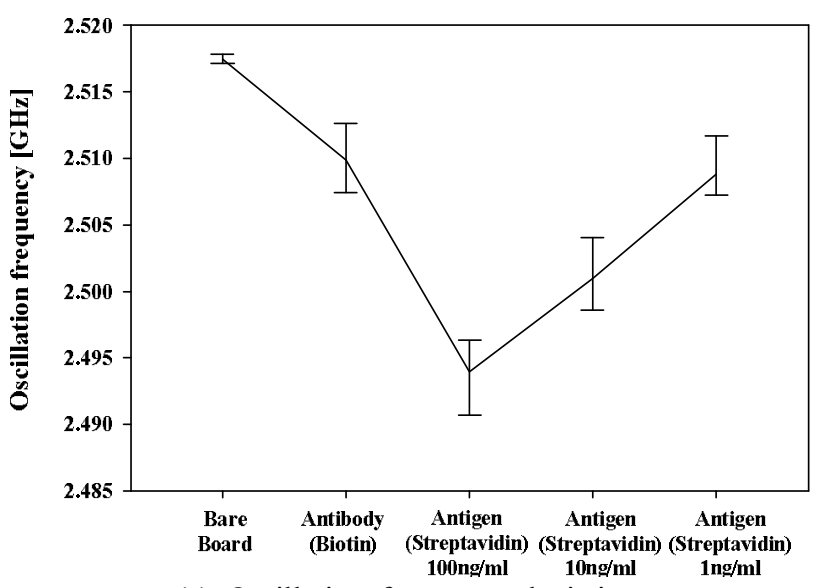

(a) Oscillation frequency deviation

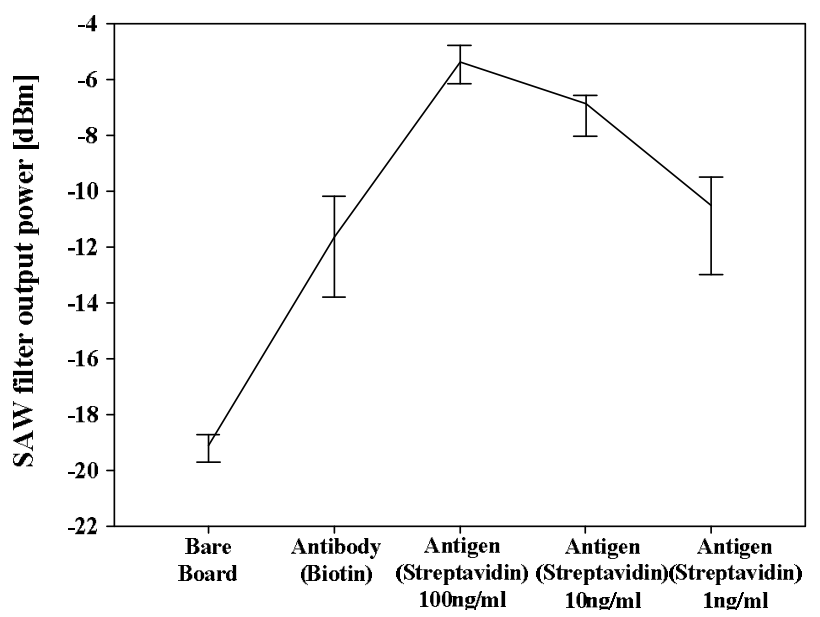

(b) SAW filter output power variation

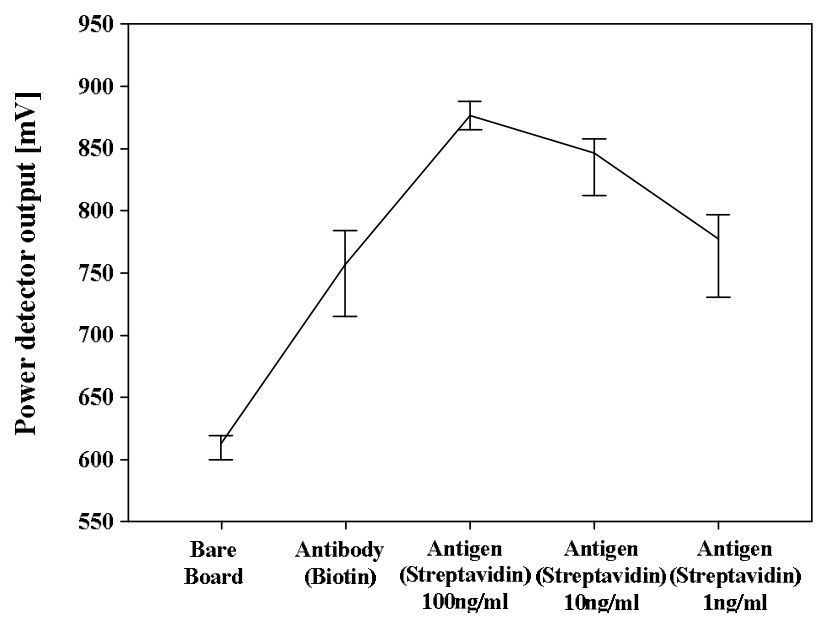

(c) Power detector output DC voltage variation

Fig. 12. Frequency deviation, amplitude variation, and power detector output power variation caused by the immobilization of biotinylated thiols and three configurations of streptavidin concentrations.

deviation for biomolecular detection has been proposed and experimentally demonstrated at a $2.4 \mathrm{GHz}$ ISM band. The proposed biosensing system showed an almost 
linear frequency response to three streptavidin concentrations when used with the biotin immobilization system. Also, it was found that the limit of detection was about $1 \mathrm{ng} / \mathrm{ml}$, This means that the system contains a highly sensitive RF biosensing device. Furthermore, the proposed device is cost-efficient and is also a simple and direct biosensing scheme with a resonator-based RF active system that does not require complex or sophisticated equipment. From the results obtained, it is clearly shown that the proposed RF biosensing system is a very powerful candidate for a wireless biosensing node in bio/medical applications.

This research was supported by the MKE(The Ministry of Knowledge Economy), Korea, under the ITRC(Information Technology Research Center) support program supervised by the NIPA(National IT Industry Promotion Agency)" (NIPA-2012-H030112-1001).

\section{References}

[1] Y. I. Kim, T. S. Park, J. H. Kang, M. C. Lee, J. T. Kim, J. H. Park, and H. K. Baik, "Biosensors for label free detection based on RF and MEMS technology," Sensors and Actuators B: Chemical, vol. 119, pp. 592-599, Dec. 2006.

[2] R. H. Farahi, T. L. Ferrell, A. Guiseppi-Elie, and P. Hansen, "Integrated electronics platforms for wireless implantable biosensors," IEEE NIH Life Science Systems and Applications Workshop (LISA), pp. 27-30, 2007.

[3] H. J. Lee, J. G. Yook, "Biosensing using split-ring resonators at microwave regime," Applied Physics Letters, vol. 92, no. 25, pp. 254103(1-3), Jun. 2008.

[4] H. J. Lee, H. S. Lee, H. H. Choi, K. H. Yoo, and J. G. Yook, "An RF circuit model for interdigital capacitor-based carbon nanotube biosensors," IEEE Transactions on Nanotechnology, vol. 9, no. 6, pp. 682-686, Nov. 2010.

[5] H. J. Lee, H. S. Lee, K. H. Yoo, and J. G. Yook, "DNA sensing using split-ring resonator alone at microwave regime," Journal of Applied Physics, vol. 108, no. 1, pp. 014908(1-6), Jul. 2010.

[6] H. J. Lee, H. S. Lee, K. H. Yoo, and J. G. Yook,
"A study on biomaterial detection using singlewalled carbon nanotube based on interdigital capacitors," Journal of Korean Insittutue of Electromangetic Engineering and Science, vol. 19, no. 8, pp. 891-898, Aug. 2008.

[7] C. Dalmay, A. Pothier, M. Cheray, F. Lalloue, M. O. Jauberteau, and P. Blondy, "Label-free RF biosensors for human cell dielectric spectscopy," International Journal of Microwave and Wireless Technologies, vol. 1, no. 6, pp. 497-504, Jan. 2010.

[8] C. Dalmay, M. Cheray, A. Pothier, F. Lalloue, M. O. Jauberteau, and P. Blondy, "Ultra-sensitive biosensor based on impedance spectroscopy at microwave frequencies for cell scale analysis," Sensors and Actuator A: Physics, vol. 162, no. 2, pp. 189197, Aug. 2010.

[9] K. Grenier, D. Dubuc, P. E. Poleni, M. Jumemura, H. Toshiyoshi, T. Fujii, and H. Fujita, "Resonant based microwave biosensors for biological cells discrimination," IEEE Radio and Wireless Symposium, pp. 523-526, Jan. 2010.

[10] Jeremy Everard, Fundamentals of RF Circuit Design with Low Noise Oscillator, John Wiley \& Sons, Ltd., 2001.

[11] F. Giannini, G. Leuzzi, Nonlinear Microwave Circuit Design, John Wiley \& Sons, Ltd., 2004.

[12] M. Regis, O. Llopis, and J. Graffeuil, "Nonlinear modeling and design of bipolar transistors ultralow phase-noise dielectric-resonator oscillators," $I E$ EE Trans. on Microwave Theory and Tech., vol. 46, no. 10, pp. 1589-1593, Oct. 1998.

[13] M. Seifert, M. T. Rinke, and H. J. Galla, "Characterization of streptavidin binding to biotinylated, binary self-assembled thiol monolayers-influence of component ratio and solvent," Langmuir, vol. 26, no. 9, pp. 6386-6393, Feb. 2010.

[14] L. Haeussling, H. Ringdorf, F. J. Schmitt, and W. Knoll, "Biotin-functionalized self-assembled monolayers on gold: surface plasmon optical studies of specific recognition reactions," Langmuir, vol. 7, no. 9, pp. 1837-1840, Sep. 1991.

[15] C. M. Pradier, M. Salmain, L. Zheng, G. Jaouen, "Specific binding of avidin to biotin immobilised on modified gold surface: Fourier transform infrared reflection absorption spectroscopy analysis," Surface Science, vol. 502-503, no. 10, pp. 193-202, Apr. 2002. 


\section{Sang-Gyu Kim}

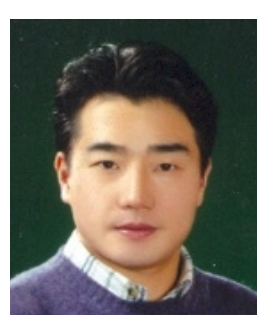

was born in Seoul, Korea. He received the B.S. and M.S. degrees in electrical and electronic engineering from Kyungwon University, Korea in 2001, 2003, respectively, and is currently pursuing the Ph.D. degree in electrical and electronic engineering at the Yonsei University, Seoul, Korea. From December 2005 to December 2008, he worked in Analog Devices Inc., Korea, as a Packaging Design Engineer. His research interests are theoretical/numerical electromagnetic modeling, characterization of microwave circuit, analysis and optimization of high-speed interconnection, including electromagnetic interference (EMI)/ electromagnetic compatibility (EMC), and remote wireless vital signal monitoring sensors.

\section{Hee-Jo Lee}

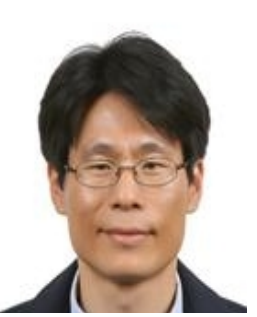

was born in Namhae, Gyeongnam, Korea. He received the Ph.D. degree in electrical and electronic engineering from Yonsei University, Korea in 2010. He is currently a Research Professor with the mechanical engineering at the Yonsei University, Seoul, Korea. From March 2010 to March 2012, he worked in electrical and electronic engineering, Yonsei University and in Graphene Research Institute, Sejong University, as a Post-Doc. Researcher. His main research interests are in the areas of electromagnetic wave and field theory, RF circuit modeling and characterization of carbon nanomaterials, including carbon nanotubes (CNTs), graphenes, and graphene oxide, carbon nanomaterial-resonator-based RF biosensors, metamaterial-based RF biosensors, microfluidic-integrated RF circuit for circulating tumor cell (CTC) detection.

\section{Jong-Gwan Yook}

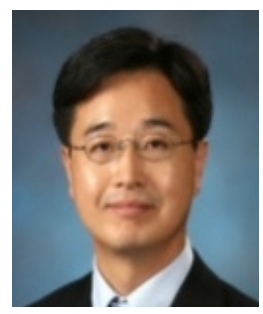

(S'89 M'97) was born in Seoul, Korea. He received the B.S. and M.S. degrees in electronics engineering from Yonsei University, Seoul, Korea, in 1987 and 1989, respectively, and the Ph.D. degree from The University of Michigan, Ann Arbor, MI, in 1996. He is currently a Professor with the School of Electrical and Electronic Engineering, Yonsei University. His main research interests are in the areas of theoretical/numerical electromagnetic modeling and characterization of microwave/millimeterwave circuits and components, design of radio frequency integrated circuits (RFIC) and monolithic microwave integrated-circuit (MMIC), and analysis and optimization of high-frequency high-speed interconnects, including signal/power integrity (EMI/EMC), based on frequency as well as time-domain full-wave methods. Recently, his research team is developing various biosensors, such as carbon-nano-tube RF biosensor for nanometer size antigen-antibody detection as well as remote wireless vital signal monitoring sensors. 